\title{
Brief Words from the Editor-in-Chief
}

Fernando Pestana da Costa, Editor-in-Chief of the EMS Newsletter

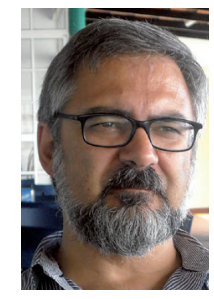

As you have read in the Message from the President, this will be the final issue of the Newsletter. From 2021 onwards it will be renamed the EMS Magazine, and, among other changes, it will boast a completely new design and layout, moving the presently existing news section to the website and switching to an "online first" publishing format.

Thus, the present issue will be the last in a series. It will also be the final issue in which Dierk Schleicher and
Jean-Paul Allouche participate as editors, both of whom will have completed their second four-year term as editors on the last day of 2020. I am grateful to them both for their highly valued collaboration and active involvement with the Newsletter over the past eight years.

Another collaborator who will cease her work with the Newsletter is EMS Press Head of Production Sylvia Fellmann Lotrovsky, who will be retiring from EMS Press in the spring and whose involvement with the Newsletter will cease with this last issue in its present form. Sylvia is a very knowledgeable and friendly person, and has 
always been available to help the editors in the myriad of things involved in producing the Newsletter. She will be missed.

This issue will, as usual, contain many interesting articles on a variety of topics. Without diminishing other contributions, let me just highlight the following ones: a timely study about women on editorial boards of scientific and EMS journals and the first four articles of what is intended to be a series presenting the work of the ten winners of the 2020 EMS Prize, as well as the 2020 Felix Klein and the 2020 Otto Neugebauer prizes. The award ceremony was planned to have taken place at the ECM in Portoroz this summer, but had to be postponed for a year due to the pandemic. This unfortunate but unavoidable decision had the fortunate consequence of allowing us time to invite each of the prize winners to write an article about their work. All have kindly agreed and the first four, by Jack Thorne, Kaisa Matomäki, Karim Adiprasito and Phan Thành Nam, appear in the present issue. The next two issues of the EMS Magazine will publish the remaining ones. Hopefully, when we all meet in Portoroz in the Summer of 2021 for the 8ECM, we will already have had the opportunity of reading the Magazine and learning about their work, and will be able to follow the prize winners' talks with enhanced pleasure. 